\title{
Heart failure with mid-range ejection fraction: Current evidence and uncertainties
}

\author{
Raffaele Martone ${ }^{1}$, Niccolò Marchionni ${ }^{1}$, Francesco Cappelli ${ }^{2,3}$ \\ ${ }^{1}$ Cardiovascular and Thoracic Department, Careggi University Hospital, Florence; ${ }^{2}$ Interventional Structural \\ Cardiology Division, Careggi University Hospital, Florence; ${ }^{3}$ Tuscan Regional Amyloid Center, Careggi University \\ Hospital, Florence, Italy
}

\begin{abstract}
Heart failure (HF) with mid-range ejection fraction (HFmrEF) has been conceptualized by the European Society of Cardiology guidelines with the aim of stimulating research to fill a gap in knowledge: whether such a condition exists as a distinct pathophysiological and clinical entity, or it is just a residual category of ejection fraction indeed is still a matter of debate. Current evidence suggests that HFmrEF represents up to one fifth of patients with HF, who may ultimately result in an intermediate clinical phenotype, as for age and gender, with an intermediate prevalence of comorbidities. Nevertheless, a strong connection exists with HF with reduced ejection fraction, since ischemic aetiology is common in both categories, conveying relevant implications for prognosis and therapeutic response. Little is known about its pathophysiology: mild systolic impairment may be not enough and advocating diastolic dysfunction may be an oversimplification. An increasing amount of data is clarifying how many of HFmrEF patients are the results of deteriorating or recovering hearts, thus underscoring that aetiology may be, more than EF, the key to understand this new category. Sparse evidence points toward a potential benefit of common HF therapies in those patients, but further research is still needed.
\end{abstract}

Correspondence: Francesco Cappelli, Intensive Cardiac Care Unit, Department of Heart Lung and Vessels, Careggi University Hospital, Largo Brambilla 3, 50134 Florence, Italy.

E-mail: cappellifrancesco@inwind.it

Key words: Heart failure; ejection fraction; mid-range.

Received for publication: 3 January 2019.

Accepted for publication: 27 March 2019.

CCopyright R. Martone et al., 2019

Licensee PAGEPress, Italy

Monaldi Archives for Chest Disease 2019; 89:1024

doi: 10.4081/monaldi.2019.1024

This article is distributed under the terms of the Creative Commons Attribution Noncommercial License (by-nc 4.0) which permits any noncommercial use, distribution, and reproduction in any medium, provided the original author(s) and source are credited.

\section{Introduction}

Heart failure (HF) has been historically categorized by left ventricular ejection fraction (LVEF) values due to prognostic and therapeutic implications, since most available interventions have been able to reduce morbidity and mortality in those with LVEF $<35-40 \%$. Beside this well-known group of patients with severe systolic dysfunction, another category has gained scientific interest: patients with HF symptoms, whose LVEF is preserved (i.e., $>50 \%$ ), but with evidence of cardiac abnormalities. Heart failure with preserved LVEF (HFpEF) is currently topic of deep research, and although an abnormal diastolic pattern has been advocated as a major pathophysiological mechanism, whether it should be looked as single disease or as multiple diseases with a common underlying physiology, is still debated [1]. Between the two, a gap has been left: patients whose EF is not severely impaired (or, at least, not below the cut-off commonly used in most HF intervention trials), but neither completely normal, thus not fulfilling the criteria for HFpEF.

This entity, already identified as a grey area of mild systolic dysfunction in the 2012 European Society of Cardiology (ESC) guidelines [2], later has been classified within the HF with preserved EF group by the 2013 ACCF/AHA guidelines [3]. Current ESC guidelines define HF with mid-range EF (HFmrEF) as the combination of signs and symptoms of HF with LVEF 40-49\% and markers of abnormal cardiac function or morphology: namely, increased levels of natriuretic peptides and at least one between left atrial enlargement and/or left ventricular hypertrophy, or left ventricle diastolic dysfunction [4]. Stuck in the middle, HFmrEF has eventually gained a formal recognition, but there are still large gaps in understanding its epidemiology, aetiology, clinical phenotype, prognosis and potential treatments.

In this brief narrative review, we will focus on what is currently known about HFmrEF, focusing on controversies and promising fields of research.

\section{Epidemiology and clinical profile}

A recent population-based analysis of the Swedish Heart Failure Registry [5] found that HFmrEF accounts for up to onefifth $(21 \%)$ of HF patients. When comparing clinical profiles across different EF ranges, HFmrEF proved to be a middle-earth as for age, female gender, comorbidities (hypertension, atrial fibrillation, lung disease), with all these features being more prevalent than in HFrEF and quite close to that found in HFpEF, with 
the notable exception of diabetes, which was significantly more prevalent in HFpEF. Where HFmrEF appeared to resemble HFrEF was in prevalence of ischemic heart disease (IHD), which also portended for both conditions a higher 1- and 3-year risk of death; moreover, previous myocardial infarction and revascularisation were more common in HFrEF and HFmrEF than in HFpEF [6]. Such a peculiarity is consistent with previous reports, suggesting that IHD may be an aetiological link between HFrEF and HFmrEF. A similar picture of HFmrEF derived from another wide population-based registry from Japan [7], where HFmrEF resulted more closely similar to HFpEF; prevalence was $17 \%$, confirming that a significant proportion of symptomatic, real world, HF patients falls into the mid category. Bhambhani et al. [8], in their work on incident HF in a pooled population from four community-based longitudinal cohorts, found an incidence of 6.7 cases per 10.000 personyears for HFmrEF, compared to 26.9 and 34.9 cases for HFpEF and HFrEF respectively. Differences in the prevalence and incidence of HF categories may still depend on variable modalities of enrolment and settings of patient management: HFpEF incidence was higher in a community-based studies, and a bimodal distribution was more typical in hospital settings, compared to the unimodal distribution usually found across different ranges of EF in outpatient studies.

\section{Outcomes}

In the Swedish Heart Failure [5] registry, all-cause mortality was similar in HFmrEF and HFpEF after adjustment for age and other confounders, and overall slightly less than in HFrEF, up to 3 years follow-up. Tsuji and colleagues [7] explored multiple prognostic outcomes and found that incidence of HF hospitalization was intermediate between HFpEF and HFrEF and all-cause and cardiovascular mortality was closer to $\mathrm{HFpEF}$, while no significant differences were seen for non-cardiovascular mortality, acute myocardial infarction or stroke among the three groups. They also found that prognostic factors identified for HFmrEF (age $>75$ years, increased BNP, diuretics use, stroke and cancer) were shared by HFpEF and HFrEF (with the notable exception of cancer and diuretics use, the latter being shared only with HFrEF). Bhambhani et al. [8] reported that all-cause mortality of HFmrEF was worse than that of HFpEF (50 vs 39 events per 1000 person-years) but comparable to that of HFrEF. The ESC Heart Failure Long-Term Registry [9] showed that all-cause, one-year mortality was $8.8 \%$ for HFrEF, 7.6\% for HFmrEF and 6.4\% in HFpEF (with a significant difference only between HFpEF and HFrEF). As a whole, available studies report slightly different prognostic profiles for HFmrEF, with a trend suggesting a truly intermediate mid-term but a better long-term prognosis, closer to that of HFpEF.

\section{Aetiology and pathophysiology}

As reported, prevalence of IHD appears to be higher in HFmrEF than in HFpEF. Such a finding is one of the most striking and consistent across different observations. After adjusting for possible confounders, Authors from the Swedish Registry [6] confirmed the meaningful asymmetric distribution of IHD among different EF ranges - namely $60 \%$ for $\mathrm{HFrEF}, 61 \%$ for $\mathrm{HFmrEF}$ and $52 \%$ for HFpEF (RR preserved $v$ s midrange: 0.91 ). Tsuji et al. [7] and Chioncel et al. [9] also reported about other aetiologies. When compared to HFpEF, HFmrEF had an intermediate prevalence of Hypertensive Heart Disease (HHD) in both studies (14.3\% $v$. $24.5 \%$, and $9.6 \%$ vs $18.1 \%$ respectively), but was more commonly related to Dilated Cardiomiopathy (DCM) $(20.3 \%$ vs $6.4 \%$, and $27.6 \%$ vs $11.6 \%$ ), and less commonly to Valve Heart Disease (VHD) (10\% vs $19.5 \%$ and $5.9 \%$ vs $14.4 \%$ ).

Thus, HFmrEF again appears to stand somehow closer to HFrEF (Table 1). Whether the two phenotypes share similarities also for pathophysiology, is less clear. Large amount of data has proven the paradigm of neurohormonal activation as a key concept in the interpretation of progression of HFrEF, at the same time providing founding elements for a rational therapeutic strategy. Currently, less is known about relative contribution of mild systolic impairment over diastolic dysfunction in HFmrEF. However, conventional categories can still offer interesting insights into problems, as proven by evaluation of temporal changes in EF and transitions through different ranges. In 3480 patients with a median follow-up of 3 years, Tsuji et al. [7] found that $21 \%$ with HFmrEF transitioned to $\mathrm{HFrEF}$ and $45 \%$ to $\mathrm{HFpEF}$; HFrEF transitioned in $21 \%$ of cases to HFmrEF and in $26 \%$ to HFpEF; conversely, only $8 \%$ of patients with HFpEF showed a downward transition. Interestingly, IHD was the most relevant independent predictor of EF worsening over time, for both HFmrEF and HFrEF: again, IHD is therefore identified as the common load dragging HFmrEF toward a worse prognosis. Of patients with HFmrEF from the study by Rastogi et al. [10], 73\% had previously had an EF $<40 \%$ and $17 \%$ an $\mathrm{EF}>50 \%$; furthermore, Authors noted that among

Table 1. Prevalence of different aetiologies across ranges of ejection fractions.

\begin{tabular}{|c|c|c|c|c|}
\hline Aetiology & & d prevale & & Reference \\
\hline & HFpEF & HFmrEF & HFrEF & \\
\hline Ischemic heart disease & 52.4 & 60.7 & 60 & Vedin et al. [6] \\
\hline & 23.7 & 41.8 & 48.6 & Chioncel et al. [9] \\
\hline & 44.1 & 52.9 & 50.1 & Tsuji et al. [7] \\
\hline Valvular heart disease & 14.4 & 5.9 & 4.4 & Tsuji et al. [7] \\
\hline & 19.5 & 10 & 4.4 & Chioncel et al. [9] \\
\hline Hypertensive heart disease & 18.1 & 9.6 & 4.5 & Chioncel et al. [9] \\
\hline & 24.5 & 14.3 & 9.3 & Tsuji et al. [7] \\
\hline Dilated cardiomyopathy & 6.4 & 20.3 & 32.2 & Tsuji et al. [7] \\
\hline & 11.6 & 27.6 & 35.1 & Chioncel et al. [9] \\
\hline
\end{tabular}

$\mathrm{HFPEF}$, heart failure with preserved ejection fraction; HFmrEF; heart failure with mid-range ejection fraction; HFrEF, heart failure with reduced ejection fraction. 
patients with HFmrEF as a result of a deteriorating, previously higher EF, diastolic dysfunction was more prevalent than among those with improving EF.

In both studies, an improving EF was associated with better prognosis. Lupon et al. [11] recently reported a prospective, observational study of the dynamic trajectories of $\mathrm{EF}$ in 3160 patients with HFrEF and HFmrEF over a 15-year follow-up. They found an overall trend towards a marked rise in EF during the first year, with a plateau up to a decade and a subsequent decline; such an inverted, U-shaped curve was more evident in those with non-ischemic aetiology and in women. Patients with HFmrEF had a less pronounced EF increase ( $3 \pm 9 \%)$ than those with HFrEF $(9 \pm 12 \%)$. Such observations suggest an intrinsic difference between HFrEF and HFpEF, with HFmrEF being at the same time a transitional grey zone and a category with inherent heterogeneity in pathophysiology.

\section{Therapeutic options}

Trends in drug prescription among patients with HFmrEF in current series [5,7] suggest either that this condition commonly represents a recovered $\mathrm{HFrEF}$ with residual disease-specific therapy or that clinicians believe that neurohormonal blockade may be useful whenever a mild systolic impairment is present. Whether such an approach is rational, is unclear. In the CHART-2 [7], betablockers were found to reduce all-cause death in HFmrEF and HFrEF (HR 0.57, $\mathrm{p}=0.010$ and $0.59, \mathrm{p}=0.008$ ), respectively), but not in HFpEF. Beneficial effect on 1-year all-cause mortality was found also by Koh et al. [9], but within the narrower boundaries of IHD for HFmrEF (HR 0.74, p=0.01 with IHD vs 0.99 without IHD); surprisingly, a reverse benefit association was found for HFpEF (HR 0.97, $\mathrm{p}=\mathrm{ns}$ vs 0.81 without IHD).

An individual patient-level meta-analysis on the efficacy of beta-blockers [12] (including 575 patients with EF 40-49\%) found a reduction in mortality compared to placebo across all EF ranges in sinus rhythm, except for those with $\mathrm{EF}>50 \%$; cardiovascular mortality was significantly reduced in HFmrEF (HR: 0.48 , CI $95 \%=$ 0.24-0.97). Beta-blockers also increased EF at 1-year follow-up in patients with baseline $\mathrm{EF}<50 \%$, with smaller increase for HFmrEF and for those of ischemic aetiology. Results from the CHARM [13] trial across the whole EF spectrum, with limitations inherent to posthoc analysis, suggest a reduction in the primary composite end point (cardiovascular death and HF hospitalization) in those treated with the Angiotensin Receptor Blocker (ARB) candesartan, both in patient with EF $\leq 40 \%$ and $40-49 \%$ (HR $0.82, p<0.001$, and 0.76 , $\mathrm{p}=0.02$, respectively), but not in patient with $\mathrm{HFpEF}$.

Of note, $\mathrm{p}$ for interaction was not significant, thus limiting translational outlooks to hypothesis generation. In the TOPCAT [14] trial, aimed at investigating the effects of spironolactone in patients with symptomatic $\mathrm{HF}$ and $\mathrm{EF} \geq 45 \%$, this agent reduced the combined end-point of cardiovascular death, HF hospitalization or aborted cardiac arrest at the lower end of the explored EF ranges $(45-50 \%)$, but had no protective effect in patients with $\mathrm{EF} \approx 60 \%$.

Cardiac rehabilitation provides a multidisciplinary frame for both educational and exercise training purposes in patients with HFmrEF: not only it can address specific needs of the patients, as exercise intolerance is a major complaint, but also allows active counselling across steady and/or transitional phases. Inclusion in rehabilitation programmes may thus be of high value in the continuum of care.

We are still far from having clear therapeutic indications for
HFmrEF. Current ESC guidelines recommend screening patients for comorbidities and prescribing diuretics to alleviate symptoms and signs of heart failure [4]. Evidence suggests that therapeutic response to available drugs in HFmrEF is closer to HFrEF, with aetiology being a possible relevant issue in predicting such response.

\section{Conclusions}

HFmrEF was originated as a residual entity: there was not a group of patients needing to be classified, but just a gap left unattended between two different HF categories (HFrEF and HFpEF). Much has been questioned about $\mathrm{EF}$ as a predictive measure. However, current clinical practice suggests that, despite its many conceptual and technical limitations, EF provides information that are useful in HF management. Yet, it is clear what EF definitely cannot do, i.e. identifying in itself a homogeneous group of patients. HFmrEF is the case, since it is evident that an EF range as narrow as absolute 10 percent is of limited utility in discriminating patients with a peculiar pathophysiology. Moreover, accuracy in EF measurement may itself be a major limitation, since interobserver variability of echocardiography in untrained professionals may be wider than that range [15].

Overall, HFmrEF appears seems to be a transition category: current observations have underscored that EF progression varies with aetiology, time from onset and gender and that the path away from - or back to - lower EF commonly passes through HFmrEF. As such, a middle category may be useful just as long as it is the occasion to make a question about past and future of a failing heart - what brings a heart to recover or to further decline - with the aim of helping clinicians understand the complexity that is hidden behind a "border line" patient.

\section{References}

1. Kishan SP, Kavita S, Mona F, et al. Heart failure with preserved ejection fraction expert panel report. JACC Heart Fail 2018;6:619-32.

2. McMurray JJ, Adamopoulos S, Anker SD, et al. ESC Guidelines for the diagnosis and treatment of acute and chronic heart failure 2012: The Task Force for the Diagnosis and Treatment of Acute and Chronic Heart Failure 2012 of the European Society of Cardiology. Developed in collaboration with the Heart Failure Association (HFA) of the ESC. Eur J Heart Fail 2012;14:803-69.

3. Yancy CW, Jessup M, Bozkurt B, et al. 2013 ACCF/AHA guideline for the management of heart failure: a report of the American College of Cardiology Foundation/American Heart Association Task Force on Practice Guidelines. J Am Coll Cardiol 2013;62:e147-e239

4. Ponikowski P, Voors AA, Anker SD, et al. 2016 ESC Guidelines for the diagnosis and treatment of acute and chronic heart failure: The Task Force for the diagnosis and treatment of acute and chronic heart failure of the European Society of Cardiology (ESC). Developed with the special contribution of the Heart Failure Association (HFA) of the ESC. Eur Heart J 2016;37:2129-200.

5. Koh AS, Tay WT, Teng TH, et al.A comprehensive population based characterization of heart failure with mid-range ejection fraction. Eur J Heart Fail 2017;19:1624-34. 
6. Vedin O, Lam CS, Koh AS, et al. Significance of ischemic heart disease in patients with heart failure and preserved,midrange, and reduced ejection fraction: a nationwide cohort study. Circ Heart Fail 2017;10:e003875.

7. Tsuji K, Sakata Y, Nochioka K, et al. Characterization of heart failure patients with mid-range left ventricular ejection fraction - a report from the CHART-2 Study. Eur J Heart Fail 2017;19:1258-69

8. Bhambhani V, Kizer JR, Lima JA, et al. Predictors and outcomes of heart failure with mid-range ejection fraction. Eur $\mathrm{J}$ Heart Fail 2018;20:651-9.

9. Chioncel O, Lainscak M, Seferovic PM, et al. Epidemiology and one year outcomes in patients with chronic heart failure and preserved, mid-range and reduced ejection fraction: an analysis of the ESC Heart Failure Long-Term Registry. Eur J Heart Fail 2017;19:1574-85.

10. Rastogi A, Novak E, Platts AE, Mann DL. Epidemiology, pathophysiology and clinical outcomes for heart failure patients with a mid-range ejection fraction. Eur J Heart Fail 2017;19:1597-05.
11. Lupón J, Gavidia-Bovadilla G, Ferrer E, et al. Trajectories of left ventricular ejection fraction in heart failure. J Am Coll Cardiol 2018;72:591-601.

12. Cleland JCF, Bunting KV, Flather MD, et al. Beta-blockers in Heart Failure Collaborative Group; Beta-blockers for heart failure with reduced, mid-range, and preserved ejection fraction: an individual patient-level analysis of double-blind randomized trials. Eur Heart 2018;39:26-35.

13. Lund LH, Claggett B, LiuJ, et al. Heart failure with mid-range ejection fraction in CHARM: characteristics, outcomes and effect of candesartan across the entire ejection fraction spectrum. Eur J Heart Fail 2018;20:1230-39.

14. Solomon SD, Claggett B, Lewis EF, et al. Influence of ejection fraction on outcomes and efficacy of spironolactone in patients with heart failure with preserved ejection fraction. Eur Heart J 2016;37:455-62.

15. McGowan JH, Cleland JG. Reliability of reporting left ventricular systolic function by echocardiography: a systematic review of 3 methods. Am Heart J 2003;146:388-97. 